\title{
EFFECTS OF INTEGRATED WEED MANAGEMENT AND BALANCED FERTILIZATION ON CROP WEEDS COMPETITION, IN CORIANDER (CORIANDRUM SATIVUM L.)
}

\author{
RAJ KUMAR NAGAR \\ Department of Agronomy, Rajasthan College of Agriculture, Maharana Pratap \\ University of Agriculture and Technology, Udaipur, Rajasthan, India
}

\begin{abstract}
A field experiment was conducted at Instructional Farm, Rajasthan College of Agriculture, Udaipur (Rajasthan), during 2002-03 and 2003-04. Pooled analysis of data indicated that all weed management practices significantly reduced the weed density and weed dry matter at all stages of crop growth period and improve yields (seed and straw yield) of crop as compared to control. Two HW at 30 and 45 DAS resulted in maximum reduction in weed density and dry matter and gave the highest seed (15.84 q/ha) and straw yield (25.27 q/ha) of coriander. However, this treatment was found at par with pendimethalin $1.0 \mathrm{~kg} / \mathrm{ha}+$ one hand weeding at $45 \mathrm{DAS}$ in reduction of weed dry matter and improving crop yields. Balanced fertilization with $60 \mathrm{~kg} \mathrm{~N}+30 \mathrm{~kg} \mathrm{P}+30 \mathrm{~kg} \mathrm{~K}+30 \mathrm{~kg}$ S/ha significantly improved weed dry matter over $N+P$ and $N+P+K$ application but also significantly improved crop yields. However, all fertilization treatments fail to affect weed density significantly.

KEYWORDS: Higher Productivity Levels, Our Nutrient Management, Crop Weed Competition in Coriander \& Evaluate the Impact of Weed Management Practices
\end{abstract}

Received: Jul 18, 2017; Accepted: Aug 03, 2017; Published: Aug 11, 2017; Paper Id.: IJASRAUG201793

\section{INTRODUCTION}

Among the spices, coriander (Coriandrum sativum L.) is cultivated in India in about 4.33 lakh ha and produces 31.8 lakh tones of seed. It is cultivated in Rabi season as a commercial crop in many parts of country. Weed control and balanced fertilization are important constraints in bumper harvest of this crop. Coriander is grown as an irrigated crop and suffered badly weed infestation. Moreover, coriander seed takes longer time for germination and also having slow early vegetative growth, the crop is very sensitive to early weed competition. Uncontrolled weeds can reduce coriander seed yield by as much as $40 \%$ (Kothari et al, 1989). The use of herbicides is one of the alternatives to eliminate crop weed competition easily. To have higher productivity levels, our nutrient management strategy are also equally important. Therefore, a study was conducted to find out the effect of weed management and balance fertilization on crop weed competition in coriander.

\section{METHODOLOGY}

A field experiment was conducted at Instructional Farm, RCA, and Udaipur during Rabi seasons of 2002-03 and 2003-04 on clay loam soil to evaluate the impact of weed management practices and balanced fertilization on weeds and yield of coriander. The experiment consisted of 11 weed management practices in main plots [weedy check, one hand weeding (HW) at $30 \mathrm{DAS}$, two $\mathrm{HW}$ at 30 and $45 \mathrm{DAS}$, pendimethalin $1.0 \mathrm{~kg} / \mathrm{ha}$, 
oxyfluorfen $0.25 \mathrm{~kg} / \mathrm{ha}$, metribuzin $0.30 \mathrm{~kg} / \mathrm{ha}$, oxadiargyl $75 \mathrm{~g} / \mathrm{ha}$, pendimethalin $1.0 \mathrm{~kg} / \mathrm{ha}+\mathrm{HW}$ at 45 DAS, oxyfluorfen $0.25 \mathrm{~kg} / \mathrm{ha}+\mathrm{HW}$ at $45 \mathrm{DAS}$, metribuzin $0.30 \mathrm{~kg} / \mathrm{ha}+\mathrm{HW}$ at 45 and oxadiargyl $75 \mathrm{~g} / \mathrm{ha}+\mathrm{HW}$ at 45 DAS] and 3 balanced fertilization treatments in sub plots $\left(\mathrm{N}_{60}+\mathrm{P}_{30}, \mathrm{~N}_{60}+\mathrm{P}_{30}+\mathrm{K}_{30}\right.$ and $\left.\mathrm{N}_{60}+\mathrm{P}_{30}+\mathrm{K}_{30}+\mathrm{S}_{30} \mathrm{~kg} / \mathrm{ha}\right)$ were laid out in a split plot design with 3 replications. All herbicide were sprayed three days after sowing using a foot sprayer fitted with a flat nozzle delivering 800 liters of water/ha. Coriander variety 'CS-6' was used as test crop. The observation on weed population, weed dry matter and coriander yield were recorded and statistically analyzed as per the standard procedure.

\section{RESULTS}

The dominant weed flora of the experimental site was Goose foot (Chenopodium murale L.), Corn spurry (Spergula arvensis L.), Indian sweet clover (Melilotus indica L.), Scarlet pimpernel (Anagallis arvensis L.) and Purple nut sedge (Cyperus rotundus L.). Among them Goose foot (Chenopodium murale L.) was found most dominant weed.

Two hands weeding at $30 \& 45$ DAS followed by pendimethalin+hand weeding was found more effective than others in reducing the weed density and dry matter throughout the crop season. Balance fertilization had no influence on weed density, while $\mathrm{N}+\mathrm{P}+\mathrm{K}$ and $\mathrm{N}+\mathrm{P}+\mathrm{K}+\mathrm{S}$ application increased weed dry matter significantly at all the stages of crop growth as compared to $\mathrm{N}+\mathrm{P}+\mathrm{K}$ fertilization. Maximum reduction in coriander seed yield was observed in the weedy check. All the weed management practices increased the seed yield of coriander significantly as compared to weedy check. The maximum seed yield (15.84 q/ha) recorded under two hand weeding was 201 per cent higher than weedy check. Amongst herbicides + hand weeding treatments, pendimethalin+hand weeding remained at par with two hands weeding, representing an increase of 199 per cent, in seed yield over weedy check. Balanced fertilization with $N+P+K+S$ significantly increased the seed yield to the tune of 18.0 and 8.90 percent over $\mathrm{N}+\mathrm{P}$ and $\mathrm{N}+\mathrm{P}+\mathrm{K}$ fertilization, respectively. Similar results on coriander with weed control and nitrogen fertilization have been reported by Patel et al (2004).

\section{CONCLUSIONS}

Pendimethalin+hand weeding at $45 \mathrm{DAS}$ with $\mathrm{N}+\mathrm{P}+\mathrm{K}+\mathrm{S}$ fertilization observed to be best treatment combination for realization of highest net returns from coriander followed by two hand weeding at 30 \& $45 \mathrm{DAS}$ with $\mathrm{N}+\mathrm{P}+\mathrm{K}+\mathrm{S}$ combination.

\section{REFERENCES}

1. Kothari, S. K.; Singh, J.P. and Singh, K.1989. Chemical weed control in Bulgarian coriander. Tropical Pest Management.35:2-5.

2. Patel, R. H.; Shroff, J.; Usadadia, V. P. and Shah, S. N. 2004. Influence of nitrogen and weed management practices on weeds and coriander. Indian Journal Weed Science.36:86-88. 


\section{APPENDICES}

Table 1: Effect of Integrated Weed Management and Balanced Fertilization on Weed Density, Dry Weight and Yield of Coriander (Pooled Data of 2 Years)

\begin{tabular}{|c|c|c|c|c|c|c|c|c|c|c|}
\hline \multirow[t]{2}{*}{ Treatments } & \multicolumn{4}{|c|}{ Weed Density $\left(\mathrm{N}_{0} / \mathrm{m} 2\right)^{*}$} & \multicolumn{4}{|c|}{ Dry Weight of Weeds (q/ha) } & \multirow{2}{*}{$\begin{array}{c}\text { Seed } \\
\text { Yield } \\
(q / h a)\end{array}$} & \multirow{2}{*}{$\begin{array}{r}\text { Straw } \\
\text { Yied } \\
\text { (G/ha) }\end{array}$} \\
\hline & $60 \mathrm{DAS}$ & $75 \mathrm{DAS}$ & $90 \mathrm{DAS}$ & At harvest & $60 \mathrm{DAS}$ & $75 \mathrm{DAS}$ & $90 \mathrm{DAS}$ & AT Harvest & & \\
\hline \multicolumn{11}{|l|}{ Weed Management } \\
\hline Weedy check & 13.81 & 15.07 & 16.36 & 17.63 & 9.16 & 13.28 & 17.45 & 22.68 & 5.27 & 8.86 \\
\hline One HW (30 DAS) & 6.35 & 8.32 & 9.08 & 9.58 & 2.23 & 2.96 & 4.38 & 6.55 & 12.31 & 20.54 \\
\hline Two HW (30 and 45 DAS) & 4.63 & 4.83 & 5.03 & 5.46 & 1.00 & 1.42 & 1.86 & 2.01 & 15.84 & 25.27 \\
\hline Pendimethalin $1.0 \mathrm{~kg} / \mathrm{ha}$ & 6.78 & 6.99 & 7.65 & 8.44 & 1.79 & 2.99 & 4.89 & 7.55 & 10.40 & 16.61 \\
\hline Oxyfluorfen $0.25 \mathrm{~kg} / \mathrm{ha}$ & 7.32 & 7.93 & 8.59 & 9.45 & 2.18 & 3.63 & 5.29 & 8.32 & 8.99 & 15.09 \\
\hline Metribuzin $0.30 \mathrm{~kg} / \mathrm{ha}$ & 9.30 & 9.80 & 10.48 & 11.18 & 3.40 & 5.06 & 6.73 & 10.96 & 8.27 & 13.28 \\
\hline Oxadiárgyl $75 \mathrm{~g} / \mathrm{ha}$ & 8.49 & 8.86 & 9.78 & 10.45 & 3.16 & 4.56 & 6.31 & 10.58 & 8.53 & 13.76 \\
\hline Pendimethalin $1.0 \mathrm{~kg} / \mathrm{ha}+\mathrm{HW}$ (45 DAS) & 5.07 & 5.24 & 5.38 & 5.83 & 1.04 & 1.48 & 1.97 & 2.69 & 15.74 & 23.88 \\
\hline Oxyfluorfen $0.25 \mathrm{~kg} / \mathrm{ha}+\mathrm{HW}$ (45 DAS) & 5.98 & 6.44 & 6.85 & 7.32 & 1.32 & 1.92 & 2.69 & 3.75 & 13.26 & 22.67 \\
\hline Metribuzin $0.30 \mathrm{~kg} / \mathrm{ha}+\mathrm{HW}$ (45 DAS) & 7.43 & 8.26 & 8.95 & 9.56 & 2.19 & 2.69 & 3.83 & 6.18 & 12.48 & 20.81 \\
\hline Oxadiạrgyl $75 \mathrm{~g} / \mathrm{ha}+\mathrm{HW}$ (45 DAS) & 6.56 & 7.22 & 7.95 & 8.67 & 1.94 & 2.44 & 3.52 & 5.82 & 12.77 & 21.06 \\
\hline $\mathrm{CD}(\mathrm{P}=0.05)$ & 0.46 & 0.46 & 0.28 & 0.33 & 0.09 & 0.32 & 0.46 & 0.71 & 0.80 & 1.42 \\
\hline \multicolumn{11}{|l|}{ Balanced Fertilization } \\
\hline $60 \mathrm{~kg} \mathrm{~N}+30 \mathrm{~kg} \mathrm{P} / \mathrm{ha}$ & 7.35 & 8.00 & 8.65 & 9.31 & 2.32 & 3.43 & 5.05 & 7.49 & 10.33 & 17.08 \\
\hline $60 \mathrm{~kg} \mathrm{~N}+30 \mathrm{kgP}+30 \mathrm{~kg} \mathrm{~K} / \mathrm{ha}$ & 7.42 & 8.08 & 8.74 & 9.42 & 2.67 & 3.87 & 5.33 & 7.97 & 11.25 & 18.31 \\
\hline $60 \mathrm{~kg} \mathrm{~N}+30 \mathrm{~kg} \mathrm{P}+30 \mathrm{~kg} \mathrm{~K}+30 \mathrm{~kg} \mathrm{~S} / \mathrm{ha}$ & 7.51 & 8.17 & 8.82 & 9.52 & 3.03 & 4.28 & 5.68 & 8.45 & 12.19 & 19.57 \\
\hline $\mathrm{CD}(\mathrm{P}=0.05)$ & NS & NS & NS & NS & 0.09 & 0.14 & 0.18 & 0.25 & 0.34 & 0.61 \\
\hline
\end{tabular}

DAS = days after sowing

Values are $\sqrt{ }(\mathbf{x}+\mathbf{0 . 5})$ transformed 
